\title{
Route to coherent supercontinuum generation in the long pulse regime
}

\author{
G. Genty \\ Tampere University of Technology, Institute of Physics, FI-33101 Tampere, Finland \\ J. M. Dudley \\ Institut FEMTO-ST, Université de Franche-Comté, 25030 Besançon, France
}

\begin{abstract}
We study numerically the possibility of generating supercontinuum with improved stability characteristics through modulation of the input pulse. General guidelines for the choice of modulation parameters leading to coherent supercontinuum generation in the long pulse regime are given.
\end{abstract}

\section{INTRODUCTION}

Supercontinuum (SC) generation in highly nonlinear and photonic crystal fibers has been the subject of intense research in the past few years, and the physical mechanisms leading to the generation of these ultra-broadband spectra are now generally well-understood [1]. Although initial research in this field tended to focus on SC generation using femtosecond pulses, much current interest is focusing on the generation of SC using picosecond (and longer) pulses as these can be generated using a variety of compact and relatively inexpensive pump sources. With such long pulses, however, the generated SC typically suffers from large shot-tovariations induced by noise-seeded modulation instability (MI) processes, and this can limit the potential application of the generated broadband spectra [2]. There is thus significant interest in developing techniques that can introduce a degree of systematic control into the shaping and stability of SC generation in this regime [3].

In this paper, we use physical arguments and numerical simulations to examine in detail the degree to which stability of the output SC spectra can be modified through a suitable input pulse modulation. Our major result is that the use of relatively large modulation strength on the input pulse can lead to a very high degree of coherence of the generated supercontinuum provided the modulation frequency and fiber length are chosen adequately. Although the use of such strongly modulated fields has been applied in previous studies of beat signal reshaping and induced modulation instability, the results here are the first that examine applications in supercontinuum spectral shaping and stability. This technique could have high impact in the spectral stabilization and control of SC generation in the long pump pulse regime.

\section{PHYSICAL GUIDELINES}

A temporal modulation is imposed across the entire pump pulse envelope in a standard way by adding to the input field a replica downshifted by $f_{\mathrm{m}}$ such that the total field can be written $A_{\mathrm{m}}(t)=A(0, t) \times\left[1+a_{0} \exp \left(\mathrm{i} 2 \pi f_{\mathrm{m}} t\right)\right]$. Here $A(0, t)=\sqrt{ } P_{0}$ $\exp \left(-2 \ln 2 \mathrm{t}^{2} / T_{\mathrm{FWHM}}{ }^{2}\right)$ is the envelope of the original pump pulse with peak power $P_{0}$ and duration $T_{\mathrm{FWHM}}$. The intensity modulation contrast (visibility) is given by $2 a_{0} /\left(1+a_{0}^{2}\right)$. This type of dual frequency field can be readily generated using optical frequency shifting techniques.

In the presence of significant modulation contrast, the input pulse can be considered as a series of ultrashort subpulses of duration $T_{\mathrm{i}} \sim 1 / 2 f_{\mathrm{m}}$ superimposed onto a broad lower amplitude background envelope. Under these conditions, the supercontinuum spectral broadening is dominated by the dynamics of these ultrashort sub-pulses, with only a minor contribution from the background envelope. We consider the case of pumping in the anomalous dispersion regime. To determine conditions on the modulation characteristics associated with coherent SC generation, we adapt previouslydeveloped criteria in the single pulse regime to the individual sub-pulses of the modulated envelope. In particular, if we consider the central sub-pulse of peak-to-background amplitude $P_{\mathrm{i}}=4 a_{0} P_{0}$, and soliton order $N_{\mathrm{i}}=\left(\gamma \mathrm{P}_{\mathrm{i}} \mathrm{T}_{\mathrm{i}} /\left|\beta_{2}\right|\right)^{1 / 2}$ we can expect coherent SC seeded by this sub-pulse provided $N_{\mathrm{i}}<N_{\mathrm{cr}}$ where $N_{\mathrm{cr}} \sim 10$ generally ensures coherent SC [1]. This condition, which applies for the case of anomalous dispersion regime pumping, can be recast into a more useful form in terms of the initial modulation frequency:

$$
f_{m} \geq \frac{\sqrt{a_{0}}}{T_{F W H M}} \cdot \frac{N}{N_{c r}},
$$

and by requiring that the propagation length is less than the fission length of the central sub-pulse, we can estimate a useful guideline for the optimal fiber length.

$$
L \approx \frac{1}{4 f_{m} \sqrt{2 \gamma a_{0} P_{0}\left|\beta_{2}\right|}} .
$$

In all of these results, $N$ is the input soliton number of the unmodulated pulse and $\beta_{2}$ and $\gamma$ represent the dispersion and nonlinearity at the pump wavelength. Although these results are based on the central sub-pulse parameters, of course neighboring sub-pulses would be expected to themselves induce similar coherent SC generation, with their presence manifested in the overall output spectrum in the form of spectral channeling at the frequency $f_{\mathrm{m}}$. 


\section{NUMERICAL MODELLING}

Although based on straightforward physical considerations, we have found using numerical simulations that the guidelines above accurately predict conditions coherent SC generation in the long pulse regime using picosecond pulses. Specifically, we consider the propagation of Gaussian $2 \mathrm{ps}, 6 \mathrm{~kW}$ peak power pulses at $1060 \mathrm{~nm}$ in a photonic crystal fiber with a zero-dispersion wavelength at $1025 \mathrm{~nm}$. The input pulse parameters correspond to a soliton number $N=177$ and under these conditions, one would expect strongly incoherent SC generation seeded by initial modulation instability (MI) dynamics. To model SC generation we use the wellestablished generalized nonlinear Schrödinger equation where our model includes quantum noise on the input pulse envelope as a one-photon-per mode background. We carry out multiple simulations using different noise seeds in order to determine average spectral characteristics and coherence properties.

Figure 1 illustrates the spectral and coherence characteristics of the generated SC after $20 \mathrm{~cm}$ in the absence of modulation on the input pulse yet in the presence of quantum noise. Clearly, the noise-seeded MI initial dynamics leads to a SC with large spectral variations from shot-to-shot and poor stability. The apparent smoothness is simply caused by the average over multiple shots.
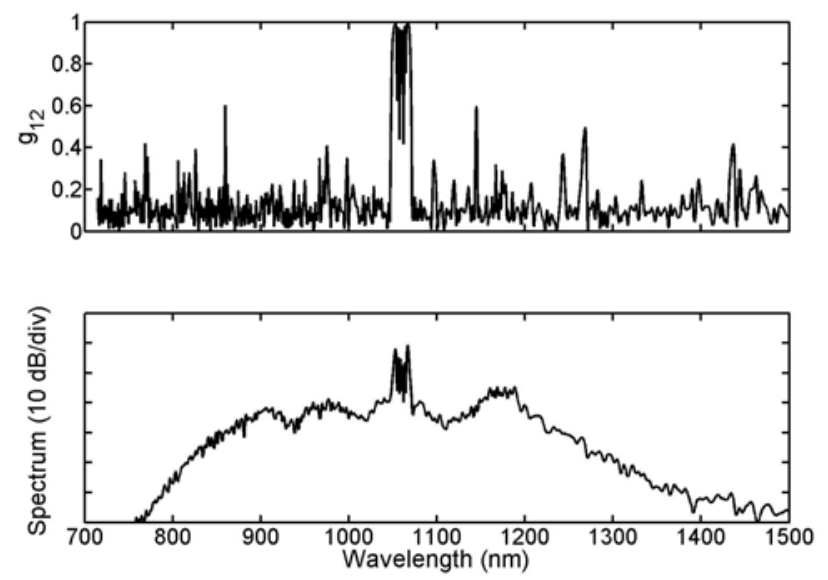

Fig. 1 Top: coherence and bottom: averaged SC spectrum generated in the unmodulated case.

We next impose a modulation on top of the input pulse with $a_{0}=0.15$ which ensures that the central sub-pulse contribution to the SC spectrum is significant. This value of $a_{0}$ gives a minimum value of $3.5 \mathrm{THz}$ for the modulation frequency for coherent SC generation. We choose $f_{\mathrm{m}}=5 \mathrm{THz}$ and a fiber length $L=12 \mathrm{~cm}$. Figure 2 shows the SC spectrum and associated coherence when the input pulse is modulated with the above characteristics. In comparison with the unmodulated pump case the SC spectrum exhibits a dramatic increase in coherence even though the overall bandwidth is comparable. This result is remarkable for the pulse duration and level of peak power envisaged here and to our knowledge represents the first example of how coherent SC can be generated in such a long pulse regime. Additional numerical simulations show that the SC coherence remains high across the SC bandwidth for longer fiber lengths exceeding several times the minimum value of $12 \mathrm{~cm}$ considered here. In general, we find that increasing $a_{0}$ improves further the stability which is expected as larger modulation contrast reduces the influence of the broad temporal background and is closer to the ideal case of a train of individual short pulses.
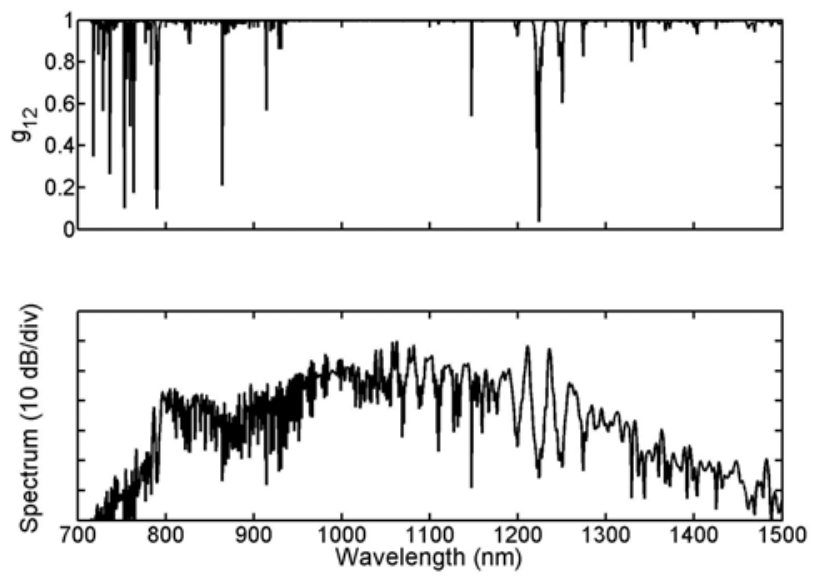

Fig. 2 Top: coherence and bottom: averaged SC spectrum generated when the input pulse is modulated.

\section{CONCLUSIONS}

We have investigated the possibility of generating broadband supercontinuum with high degree of coherence in the long pulse regime. Specifically, we have shown that imposing an initial modulation with appropriate characteristics on top of the broad pulse can lead to a fully coherent continuum which is in marked contrast with incoherent spectra generated by unmodulated pulses. In fact, the recipe given here for the generation of coherent SC in the long pulse regime is rather general and can be further applied to even much longer initial pulse duration. However, as the initial pulse duration increases, the comb-like structure of the SC becomes inherently more apparent. These findings could help solving the long-standing problem of coherent SC generation in the long pulse regime.

\section{REFERENCES}

[1] J. M. Dudley, G. Genty, S. Coen, "Supercontinuum Generation in Photonic Crystal Fiber”, Rev. Mod. Phys. 78 1135-1184 (2006)

[2] D. Solli, C. Ropers, P. Koonath, B. Jalali, "Optical rogue waves", Nature 450, 1054-1057 (2007).

[3] S. Kobtsev and S. Smirnov, "Influence of noise amplification on generation of regular short pulse trains in optical fibre pumped by intensity-modulated CW radiation”, Optics Express 16, 7427-7434 (2008). 